\title{
Macrophage engulfment of a cell or nanoparticle is regulated by unavoidable opsonization, a species-specific 'Marker of Self' CD47, and target physical properties
}

Nisha G. Sosale, Kyle R. Spinler, Cory Alvey, and Dennis E. Discher

Molecular \& Cell Biophysics Lab and NanoBioPolymers Lab

University of Pennsylvania, Philadelphia, PA 19104 
Abstract

Professional phagocytes of the mononuclear phagocyte system (MPS), especially ubiquitous macrophages, are commonly thought to engulf or not a target based strictly on 'eat me' molecules such as Antibodies. The target might be a viable 'self' cell or a drug-delivering nanoparticle, or it might be a cancer cell or a microbe. 'Marker of Self' CD47 signals into a macrophage to inhibit the acto-myosin cytoskeleton that makes engulfment efficient. In adhesion of any cell, the same machinery is generally activated by rigidity of target surfaces, and recent results confirm phagocytosis is likewise driven by the rigidity typical of microbes and many synthetics. Basic insights are already being applied to make macrophages eat cancer and to delay nanoparticle clearance for better drug delivery and imaging.

(123 words; 120 word limit)

2750 words in text; 2000 word limit

53 references; 50 reference limit 


\section{Introduction}

A macrophage is by definition a large cell that devours, with principal 'targets' for engulfment being microbes that constantly cross tissue barriers. Additional targets are now wellappreciated to include all types of injected particles, including nanoparticles, and also senescing or dead cells in the same tissue, but there are also exciting efforts to make macrophages eat cancer cells. Some features of a target can greatly influence eating by a macrophage. These features are so far understood to include surface molecules that promote eating, at least one that inhibits eating, and physical properties such as target shape and rigidity. This brief opinion article focuses particularly on recently obtained insights into molecular mechanisms of inhibition and target physical properties.

Phagocytosis is likely an ancient evolutionary development that provided sustenance to some of the first amoeboid cells. With soft plasma membranes rather than the rigid cell walls of bacteria, ancient amoeba (like modern amoeba such as Dictyostelium) could wrap around their target to engulf it and digest it within a phagosome [1]. Fast forward eons to organisms like humans that gain nutrition through a highly differentiated and multi-cellular digestive tract, and phagocytosis is a highly efficient process used only by specialized cells of the mononuclear phagocyte system (MPS). Microbes (in and on us) remain major targets as they not only out-number and outproliferate our own cells but also invade through any and all compromised tissue barriers [2]. The principal cell types of the MPS are macrophages which reside in every tissue and monocytes that circulate out of the bone marrow to enter a tissue and differentiate to macrophages $\left[3^{* *}, 4\right]$. Crucially, MPS cells as well as highly phagocytic neutrophils must - for the health of the organism - choose to devour 'foreign' targets rather than devouring human 'self' cells or extracellular matrix that generally surrounds the phagocytic cell. Phagocytosis thus evolved for engulfment and destruction of 'foreign' strictly for protection of the organism.

A variety of molecular cues and sensor assemblies must be used by our MPS cells to distinguish and destroy 'foreign' amidst 'self'. Many decades of work have elaborated a list of biochemical entities, soluble and/or surface bound, that activate macrophages (we will hereafter ignore the distinction from monocytes) to initiate engulfment of a target. One of the most important classes of molecules that is described below in context are immunoglobulin-G (IgG) antibodies which diffuse and bind to a target surface so that when a macrophage contacts the target, the constant fragment $(\mathrm{Fc})$ of the IgG binds the macrophage membrane receptor FcyR 
and (for some classes of FcyR) activates the macrophage to eat the opsonized target. Importantly, while it seems commonly presumed that our 'self' cells simply lack surface 'opsonization' by such activating molecules as lgG, it is now clear that 'self' recognition is not simply the absence of a 'foreign' signal. Instead, a dominating and passivating interaction occurs between a 'Marker of Self' CD47 membrane protein on a candidate target and the macrophage membrane receptor CD172a (also known as SIRPA, signal-regulatory protein alpha). Controlling the balance of 'eat me' cues (e.g. IgG-FcyR interaction) and 'don't eat me' signals (CD47-SIRPA) is currently an active area of translation to the clinic for anti-cancer therapy [5] and has begun to be exploited on nanoparticles in pre-clinical model [6]. However, the decision-making process within the macrophage remains a topic in need of deeper insight.

An explosion of efforts to make a broad range of injectable and implantable particles or devices for therapy and diagnostics has also revealed the MPS to be a major impediment to delivery. Make a nano-particle, inject it into the bloodstream of a mouse or man, and one invariably finds that most of the particles have been eaten by MPS cells of the spleen and of the liver (the latter are called Kupffer cells). Based on several decades of work on a diversity of nanoparticles, such clearance can be delayed but never eliminated $[7,8,9,10]$. Studies of macrophages in conventional static culture (where diffusion and buoyancy can dominate) have questioned whether uptake of small nanoparticles occurs by phagocytosis [11]. In vivo, however, bloodborne nanoparticles flow into contact with macrophages that line the spleen and liver vasculature, where these cells constantly and actively filter out senescent blood cells (e.g. after blood storage) to maintain blood homeostasis. A leaky vasculature at a site of injury or disease such as an infarct in the heart or a tumor can allow sufficiently small particles to permeate tissue and perhaps be retained [12]. However, when macrophages in damaged and disease sites are examined (too rarely), they prove to be major consumers of permeating nanoparticles [13]. Macrophages are indeed resident if distinct in every tissue in the body [ $\left.3^{\star}\right]$, and at damaged and disease sites they will at least be involved in clearing dead and dying tissue. A large implant (or even a splinter) also damages tissue and causes a 'foreign body response' that starts with serum protein deposition and soon recruits phagocytes to the site, but phagocytosis is frustrated for large implants and somehow triggers macrophage-macrophage fusion to a 'foreign body giant cell' that encases the implant [14]. Physical size is thus a factor in macrophage function, but the focus below is on targets including cells that are cell-sized or smaller, with attention to additional properties such as target shape and flexibility. 


\section{'Eat Me' signals can be weak or strong but are unavoidable}

IgG's are well-known for high affinity interactions ( nano-Molar) between epitopes and their antibody fragment (Fab) domains, but they are also large glycoproteins of $\sim 150,000$ Daltons with considerable surface area to mediate non-specific interactions. They are among the most abundant proteins in normal serum at $\sim 100 \mu \mathrm{M}$. Antibodies and other serum proteins physisorb in vivo to red blood cells (RBCs) [15,16], to viruses [17], and even to particles coated with PEG (polyethylene glycol) which otherwise delays adsorption and in vivo clearance from minutes to many hours[18]. Autologous IgG binding to autologous RBCs in humans and dogs in vivo increases up to seven-fold toward the end of the cell's $\sim 100$-day life span. Aged human RBCs also lack other "eat me" signals such as exposed phosphatidylserine[19,20]. IgG opsonization is increased in blood diseases including sickle cell anemia and thalassemia, among other conditions, where phagocytosis and cell clearance are also increased (tabulated recently in [21*]). When IgG-opsonized RBCs and particles are phagocytosed in vitro, uptake is hyperbolic and saturable versus IgG, which is consistent with specific activation of the FcyR phagocytosis pathway. Because macrophages and phagocytic dendritic cells can also function as antigen presenting cells, it seems sensible that engulfment can promote immunogenicity in vivo even to foreign polymers [22].

Binding of the IgG's Fc domain to the macrophage FcyR receptor triggers phagocytic cup formation in a coordinated process of adhesion, pseudopod extension, and eventually internalization with phagosome closure. The surface interactions initiate Src family phosphorylation of immunoreceptor tyrosine based activating motifs (ITAMs) that then propagate a phosphorylation cascade [23]. Phosphopaxillin and F-actin [24,25] accumulate in minutes or less together with other structural components at this dynamic phagocytic synapse. The process is highly analogous to adhesion formation upon integrin binding to rigid extracellular matrices, wherein the nascent adhesion matures to a focal adhesion only when the F-actin cytoskeleton is mechanically organized or pulled upon by nonmuscle myosin-II (MII) phospho-protein [26,27]. At the phagocytic synapse, MIIA accumulates to greatly help with pulling targets into a macrophage, including lgG-opsonized targets as small as $100 \mathrm{~nm}$ nanoparticles (perhaps smaller) and at least as large as opsonized RBCs $[6,28,29]$. Engulfment of such targets is greatly decreased by inhibiting MIIA motor activity with the drug blebbistatin which also blocks MII localization without affecting F-actin or phosphopaxillin. Uptake increases linearly with MII activity based on its knockdown and overexpression [28]. At least above a low 
baseline level of uptake in vitro, MII makes phaogocytosis efficient for our macrophages if not for ancient amoeba like Dictyostelium [1].

\section{CD47 mechanism of inhibition: just don't pull it in!}

As an IgG-opsonized target contacts a macrophage and adheres intimately via FcyR, the parallel presence on the target of an appropriate form of CD47 can lead to binding to the macrophage phagocytosis inhibitory receptor SIRPA, which accumulates in the synapse [29]. The latter complex somehow phosphorylates SIRPA's cytoplasmic immunoreceptor tyrosine based activating motifs (ITIMs), which activates the immunomodulatory phosphatase SHP-1 (Src homology region 2 domain-containing phosphatase-1) [30] to regulate multiple proteins by dephosphorylation [31], including deactivation of MII $[6,28]$. SIRPA-null macrophages engulf IgG-opsonized mouse RBCs more readily than wild-type macrophages [31] and show no major differences in phospho-FcyR nor the downstream effectors phospho-Syk or phosho-Cbl, which suggests that regulation of proteins even further downstream is key. Inhibition of downstream actomyosin contractility at the phagocytic synapse $[6,28]$ could indeed explain various observations that CD47 partially blocks engulfment of not only mouse RBCs - which started the entire 'Marker of Self' field [32] but also cancer cells $\left[33^{*}, 34^{* *}\right]$ and opsonized polystyrene beads (100 nm to $6 \mathrm{um}$ ) that display CD47's binding domain in parallel with IgG $[6,28]$. The effectiveness of CD47 with small nanobeads is surprising because pulling in large particles with MII forces seems more understandable than pulling in small particles.

CD47 and SIRPA arose simultaneously in amniotes, not being found even in amphibians [35], and are thus more recent inventions than the actomyosin cytoskeleton. CD47 is found on all cells in man and mouse while the expression of SIRPA is more restricted. CD47 knockout mice have one-quarter to one-half the lifespans of normal mice, and also show evidence of anti-RBC antibodies as well as anemia [36]. This is consistent with the idea that a modest level of opsonization exists in vivo which tilts the balance toward engulfment.

Saturable binding of SIRPA, CD47, and/or CD47-derived 'Self' peptide to beads as well as to living cells shows the intermediate strength interaction (sub-micro-Molar) has evolved to be largely species-specific $[6,28,37]$. The molecules also differ between strains of mice. NOD.SCID strains of mice uniquely express a SIRPA variant that binds human CD47 with similar affinity as human SIRPA, which partially explains why these are the best mouse choice for engraftment of human stem cells [38]. One recent binding study with non-glycosylated recombinant proteins by 
surface plasmon resonance reported the cross-species affinity as 10 -fold stronger than between human proteins [39], concluding that the interaction is anomalously strong. Species specificity in vivo is a critically important issue because human-specific blocking antibodies have been injected intravenously together with opsonizing $\lg G$ to impede growth and even shrink tumors of human cancer cell lines in mice. As emphasized by others $[40,41,42]$, injection of any reagent that binds human-CD47 would bind to every cell membrane in the body, even if cancer cells have several fold more of this ubiquitous protein $[43,44]$. On the other hand, CD47 is far from the most abundant protein on cells ( 250 molecules $/ \mu m^{2}$ on RBCs which is $10-20$ fold less than Glycophorin-A), so that blocking CD47 even with $\lg$ is not expected to drive strong phagocytosis unless an additional and far more abundant "eat me" cue is also on a candidate target. Such estimations should of course be put to the test by direct experiments (eg. with removal of Fc from the IgG per [28]). The half-max density for inhibition by CD47 on beads is independent of particle size and is $\sim 20$ molecules $/ \mu \mathrm{m}^{2}$, which is consistent with the minimum density of CD47 on circulating RBCs from patients with anemia [6,28]. For senescing neutrophils, CD47 is somehow down-regulated from the surface and the needed cue to drive macrophage engulfment seems to be surface-exposed calreticulin (from the endoplasmic reticulum) rather than IgG [45]. Some of the above ideas are currently being put to the test in the clinic [5] with initial results from anti-cancer clinical trials hopefully reported within a year or two. Safety is of course the first question of concern for systemic injection of any entity that limits macrophages from recognizing 'self'.

\section{Rigid cells and particles drive phagocytosis, but shape and size can frustrate} A relatively new principle in cell biology that applies to many cell types is that adhesion-induced activation of myosin-II is maximized by adhesion to a substrate that is rigid (like glass or plastic) rather than flexible like most soft tissues. Bacteria such as $E$. Coli and fungi such as yeast have cell walls as rigid as some plastics [46]. For RBCs, cell stiffness is 50-fold higher for the erythroblasts that interact with macrophages, and it is similarly higher in senescence and in diseases ranging from inherited anemias to malaria (tabulated in [21*]). With spherical microparticles made of hydrogels and opsonized by lgG, engulfment is proportional to stiffness, which was also shown to drive focal adhesion protein assembly at the synapse [47]. Stiffness changes occur with cancer cells and with chemotherapy $[48,49]$. Soft cancer cells might thus escape anticancer efforts aimed at inhibiting CD47-SIRPA interactions [34]. However, a clear relation of cell or particle stiffness to CD47 signaling and to cells with more complicated shapes had been untested until recently. 
With normal human RBCs, controlled stiffening within seconds was recently demonstrated without compromising CD47 binding to SIRPA [21*]. Despite the ability to bind, in vitro phagocytosis (in serum) of human RBCs opsonized by lgG (or not at all) was always fastest and greater in number for rigid RBCs. Rigid RBCs showed active myosin-Il at the synapse, suggesting CD47 did not signal even though it could bind SIRPA. As expected, the MII inhibitor blebbistatin blocked MII accumulation and RBC engulfment. Injection of rigid RBC discocytes into the circulation of the NOD.SCID strain of mouse also confirmed equal clearance by splenic macrophages independent of whether CD47 was blocked or not with antibody. Synthetic polymer discs resembling RBCs and that lacked any CD47 or other RBC proteins were also shown to be removed from circulating blood far more rapidly when they were stiff rather than soft [50]. Mechanistically, stiff cells and particles become stuck in narrow splenic slits [51], which could facilitate probing and clearance by splenic macrophages [52]. However, the results showing rigid RBC discocytes were engulfed independent of CD47 presented a paradox for the field in that rigid CD47-beads definitely do signal self and thereby impede engulfment both in vitro and in vivo [6].

Shape is an additional target factor that also modulates phagocytosis and resolves the apparent paradox. Polystyrene microbeads melted and distorted into diverse shapes, for example, are engulfed by macrophages more readily as spheres than as non-spheres when IgG opsonized [53]. Such findings seem relevant to the diverse shapes of bacteria and fungi, which are invariably rigid as noted above. Flexible PEG-based filaments also persist in the in vivo circulation many days longer than spherical particles of the same type of polymer, with particles always cleared, eventually, by the spleen and liver [8]. With normal human RBCs, the rapid and controlled stiffening approach $\left[21^{*}\right]$ was used to make rounded, cup-shaped RBC 'stomatocytes', and these were found to signal 'self' much better than rigid RBC discocytes. This is likely due to the discocyte's rigid concavities that could not contact and signal 'self' to the macrophage. In the same studies, native and flexible human-RBC discocytes with the requisite lgG-opsonization were seen in video-microscopy to be greatly distended by the human macrophage, with myosin-II turned off by CD47 signaling but actin polymerization driving protrusions as if pushing the cell away in recognition of 'self'. Thus, since rigid but rounded cells do signal 'self' - even if not as efficiently as flexible RBC - one can ultimately understand the success in delaying clearance of CD47-nanobeads that then enable better tumor imaging and 
drug delivery. However, it seems that a greater advantage might be achieved with flexible beads that avoid the intrinsic activation of myosin-II.

\section{Conclusions}

A deeper understanding of macrophage interactions - beyond molecular 'eat me' signals - is impacting many diverse fields. These range from cancer immunotherapy to nano-particle delivery of drugs and dyes to tumors. The finding that target rigidity favors eating means that even if microbes were to acquire CD47 from host membrane or through acquiring such a gene, the softness of our own cells provides an intrinsic advantage over rigid microbes. On the other hand, in osteoclastogenesis wherein cell fusion on bone drives 'giant cell' formation [14], we might now infer this process to be a consequence of frustrated phagocytosis on a rigid target, which over-rides any self-recognition inhibitory signals that might be present (or not) on bone matrix. Regardless, macrophages clearly calculate through diverse physicochemical signals in choosing to devour or not.

\section{Acknowledgments}

A This work was supported by the National Institutes of Health, National Institute of Biomedical Imaging and Bioengineering (grant R01-EB007049),NationalHeart, Lung, and Blood Institute (grant R01-HL124106), National Institute of Diabetes and Digestive and Kidney Diseases (grants P01-DK032094 and P30-DK090969), National Center for Advancing Translational Sciences (grant 8UL1TR000003); the National Science Foundation (Materials Research Science and Engineering Center and Nano Science and Engineering Center-Nano Bio Interface Center), and an International Research Training Group grant 1524. 


\section{References}

Papers of particular interest, published within the period of review, have been highlighted as:

* of special interest

** of outstanding interest

1. Shu S, Liu X, Korn ED: Blebbistatin and blebbistatin-inactivated myosin II inhibit myosin II-independent processes in Dictyostelium. Proc Natl Acad Sci U S A 2005, 102:1472-7.

2. Skoczek DA, Walczysko P, Horn N, Parris A, Clare S, Williams MR, Sobolewski A: Luminal microbes promote monocyte-stem cell interactions across a healthy colonic epithelium. J Immunol 2014, 193:439-451.

3. Yonit L, Deborah W, Ronnie BG, David E, Keren SH, Merad M, Jung S, Amit I: Tissueresident macrophage enhancer landscapes are shaped by the local

microenvironment. Cell 2014, 159:1312-1326.

${ }^{* *}$ Identified over 12,000 enhancers that are macrophage specific and determined gene expression profiles for seven different tissue-resident-macrophages. These expression profiles were independent of macrophage developmental origins, but dependent on environmental factors that influence chromatin landscape and thus macrophage behavior. Understanding these environmental signals could broaden macrophage immunotherapy and enhance developing therapies.

4. Gosselin D, Link VM, Romanoski CE, Fonseca GJ, Eichenfield DZ, Spann NJ, Stender JD, Chun HB, Garner H, Geissmann F, Glass CK. Environment drives selection and function of enhancers controlling tissue-specific macrophage identities. Cell 2014, 159:13271340.

5. Burgess $M$ : A phase 1, dose finding study of CC-90002, in subjects with advanced solid and hematologic cancers. NCT02367196 2015.

https://clinicaltrials.gov/ct2/show/NCT02367196?term=cd47\&rank=2

6. Rodriguez PL, Harada T, Christian DA, Pantano DA, Tsai RK, Discher DE.: Minimal "self" peptides that inhibit phagocytic clearance and enhance delivery of nanoparticles. Science 2013, 339:971-975.

7. Hu CM, Zhang L, Aryal S, Cheung C, Fang RH, Zhang L: Erythrocyte membranecamouflaged polymeric nanoparticles as a biomimetic delivery platform. Proc Natl Acad Sci U S A 2011, 108:10980-10985.

8. Geng Y, Dalhaimer P, Cai S, Tsai R, Tewari M, Minko T, Discher DE: Shape effects of filaments versus spherical particles in flow and drug delivery. Nature Nanothechnology 2007, 2:249-255. 
9. Chambers E, Mitragotri S: Long circulating nanoparticles via adhesion on red blood cells: Mechanism and extended circulation. Experimental Biology and Medicine 2007, 232:958-966.

10. Bartlett DW, Su H, Hildebrandt IJ, Weber WA, Davis ME: Impact of tumor-specific targeting on the biodistribution and efficacy of siRNA nanoparticles measured by multimodality in vivo imaging. Proc. Natl. Acad. Sci. U.S.A 2007, 104:15549.

11. Swanson JA, Hoppe AD. The coordination of signaling during Fc receptor-mediated phagocytosis. J Leukoc Biol 2004, 76:1093.

12. Wong C, Stylianopoulos T, Cui J, Martin J, Chauhan VP, Jiang W, Popovic Z, Jain RK, Bawendi MG, Fukumura D: Multistage nanoparticle delivery system for deep penetration into tumor tissue. Proc. Natl. Acad. Sci. U.S.A 2011, 108:2426-2431.

13. Turk MJ, Waters DJ, Low PS: Folate-conjugated liposomes preferentially target macrophages associated with ovarian carcinoma. Cancer Letters 2004, 213:165-172.

14. Rhee I, Davidson D, Souza CM, Vacher J, Veillette A: Macrophage Fusion Is Controlled by the Cytoplasmic Protein Tyrosine Phosphatase PTP-PEST/PTPN12. Mol Cell Biol 2013, 33:2458-2469.

15. Turrini F, Arese P, Yuan J, Low PS: Clustering of integral membrane proteins of the human erythrocyte membrane stimulates autologous IgG binding, complement deposition, and phagocytosis. J Biol Chem 1991, 266:23611-23617.

16. Franco RS, Puchulu-Campanella ME, Barber LA, Palascak MB, Joiner CH, Low PS, Cohen $\mathrm{RM}$ : Changes in the properties of normal human red blood cells during in vivo aging. Am J Hematol 2013, 88:44-51.

17. Wilflingseder D, Banki Z, Garcia E, Pruenster M, Pfister G, Muellauer B, Nikolic DS, Gassner C, Ammann CG, Dierich MP, Piguet V, Stoiber H: IgG opsonization of HIV impedes provirus formation in and infection of dendritic cells and subsequent longterm transfer to T cells. J Immunol 2007, 178:7840.

18. Discher DE, Ortiz V, Srinivas G, Klein ML, Kim Y, Christian D, Cai S, Photos P, Ahmed F. Emerging applications of polymersomes in delivery: from molecular dynamics to shrinkage of tumors. Prog Polym Sci 2007, 32:838-857. 
19. Lundqvist M, Stigler J, Elia G, Lynch I, Cedervall T, Dawson KA: Nanoparticle size and surface properties determine the protein corona with possible implications for biological impacts. Proc Natl Acad Sci U S A 2008, 105:14265.

20. Rettig MP, Low PS, Gimm JA, Mohandas N, Wang J, Christian JA: Evaluation of biochemical changes during in vivo erythrocyte senescence in the dog. Blood 1999, 93:376-384.

21. Sosale NG, Rouhiparkouhi T, Bradshaw AM, Dimova R, Lipowsky R, Discher DE: Cell rigidity and shape override CD47's "self"-signaling in phagocytosis by hyperactivating myosin-II. Blood 2015, 125:542-552.

*Through glutaraldehyde treatment of RBCs was able to selectively rigidify RBCs and show that CD47 signaling can be dominated by cell rigidity. CD47 signal can be restored by changing the shape of the RBC to a more spherical one. This explains the paradox that polymers displaying CD47 have reduced clearance. These results provide insight to clearance of old RBCs by macrophages, the role macrophages play in RBC formation, and cancer CD47 immunotherapy.

22. Armstrong JK1, Hempel G, Koling S, Chan LS, Fisher T, Meiselman HJ, Garratty G: Antibody against poly(ethylene glycol) adversely affects PEG-asparaginase therapy in acute lymphoblastic leukemia patients. Cancer 2007, 110:103-111.

23. Cox D, Greenberg S: Phagocytic signaling strategies: Fc(gamma) receptor-mediated phagocytosis as a model system. Seminars in Immuology 2001, 13:339-345.

24. Greenberg S, Chang P, Silverstein SV: Tyrosine phosphorylation of the gamma subunit of Fc gamma receptors, p72syk, and paxillin during Fc receptor-mediated phagocytosis in macrophages. J Biol Chem 1994, 269:3897 - 3902.

25. Aderem A, Underhill BM: Mechanisms of phagocytosis in macrophages. Annu Rev Immunol 1999, 17:593 - 623.

26. Wolfenson H, Bershadsky A, Henis YI, Geiger B: Actomyosin-generated tension controls the molecular kinetics of focal adhesions. J Cell Sci 2011, 124:1425-1432.

27. Raab M, Swift J, Dingal D, Shah P, Shin JW, Discher DE: Crawling from soft to stiff matrix polarizes the cytoskeleton and phosphoregulates myosin-II heavy chain. J Cell Biol 2012, 199:669-683.

28. Tsai RK, Discher DE: Inhibition of "self" engulfment through deactivation of myosin-II at the phagocytic synapse between human cells. J Cell Biol 2008, 180:989-1003.

29. Tsai RK, Rodriguez PL, Discher DE: Self inhibition of phagocytosis: The affinity of 'marker of self' CD47 for SIRP alpha dictates potency of inhibition but only at low expression levels. Blood Cells Mol Dis 2010, 45:67-74. 
30. Murata Y, Saito Y, Kaneko T, Kotani T, Kaneko Y, Ohnishi H, Matozaki T: Autoimmune animal models in the analysis of the CD47-SIRP alpha signaling pathway. Methods 2014, 65:254-259.

31. Okazawa H, Motegi S, Ohyama N, Ohnishi H, Tomizawa T, Kaneko Y, Oldenborg PA, Ishikawa $\mathrm{O}$, Matozaki $\mathrm{T}$ : Negative regulation of phagocytosis in macrophages by the CD47-SHPS-1 system. J Immunol 2005, 174:2004-2011.

32. Oldenborg PA, Zheleznyak A, Fang YF, Lagenaur CF, Gresham HD, Lindberg FP: Role of CD47 as a marker of self on red blood cells. Science 2000, 288:2051-2054.

33. Willingham SB, Volkmer JP, Gentles AJ, Sahoo D, Dalerba P, Mitra SS, Wang J, ContrerasTrujillo H, Martin R, Cohen JD, Lovelace P, Scheeren FA, Chao MP, Weiskopf K, Tang C, Volkmer AK, Naik TJ, Storm TA, Mosley AR, Edris B, Schmid SM, Sun CK, Chua MS, Murillo O, Rajendran P, Cha AC, Chin RK, Kim D, Adorno M, Raveh T, Tseng D, Jaiswal S, Enger PØ, Steinberg GK, Li G, So SK, Majeti R, Harsh GR, van de Rijn M, Teng NN, Sunwoo JB, Alizadeh AA, Clarke MF, Weissman IL: The CD47-signal regulatory protein alpha (SIRPa) interaction is a therapeutic target for human solid tumors. Proc Natl Acad Sci U S A 2012, 109:6662.

*This work expanded our clinical knowledge of CD47 expression on human tumor samples by measuring CD47 abundance and relating expression levels of CD47 to clinical outcomes. It was found that on average, over a variety of cancer types and tissue samples, CD47 expression was increased 3.3 fold compared to normal tissue types. Furthermore, higher expression of CD47 was correlated with a negative prognosis. This suggests that as cancer develops a selection process occurs for mutations that increase CD47 in order to avoid macrophage clearance. This dependence on CD47 makes it an important therapeutic target for cancer.

34. Weiskopf K, Ring AM, Ho CC, Volkmer JP, Levin AM, Volkmer AK, Ozkan E, Fernhoff NB, van de Rijn M, Weissman IL, Garcia KC: Engineered SIRPa variants as

immunotherapeutic adjuvants to anticancer antibodies. Science 2013, 341:88-91.

${ }^{* *}$ High-affinity SIRPa monomers were engineered to bind to CD47 and block 'self' signaling. This monomer was found to inhibit tumor growth similar to the monoclonal CD20 antibody Rituximab which is used in the clinic. Combination of SIRPa monomers with therapeutic antibodies (Rituximab and trastuzumab) resulted in complete inhibition of tumor growth and significantly increases survival of mice.

35. Bentley AA, Adams JC: The Evolution of Thrombospondins and Their Ligand-Binding Activities. Mol Biol and Evol 2010, 9:2187-2197.

36. Oldenborg PA, Gresham HD, Lindberg FP: CD47-signal regulatory protein a (SIRPalpha) regulates Fcgamma and complement receptor-mediated phagocytosis. J Exp Med 2001, 193:855-862. 
37. Subramanian S, Parthasarathy R, Sen S, Boder ET, Discher DE: Species- and cell typespecific interactions between CD47 and human SIRPalpha. Blood 2006, 107:2548-2556.

38. Takenaka K, Prasolava TK, Wang JCY, Mortin-Toth SM, Khalouei S, Gan OI, Dick JE, Danska JS: Polymorphism in Sirpa modulates engraftment of human hematopoietic stem cells. Nat Immunol 2007, 8:1313-1323.

39. Kwong LS, Brown MH, Barclay AN, Hatherley D: Signal-regulatory protein alpha from the NOD mouse binds human CD47 with an exceptionally high affinity - implications for engraftment of human cells. Immunology 2014, 143:61-67.

40. Soto-Pantoja DR, Miller TW, Frazier WA, Roberts DD: Inhibitory signaling through signal regulatory protein-alpha is not sufficient to explain the antitumor activities of CD47 antibodies. Proc Natl Acad Sci U S A 2012, 109:2842-2842.

41. Zhao XW, Matlung HL, Kuijpers TW, van den Berg TK: On the mechanism of CD47 targeting in cancer. Proc Natl Acad Sci U S A 2012, 109:2843-2843.

42. Willingham SB, Volkmer J-P; Weiskopf K, Ring AM, Weissman IL: Reply to Soto-Pantoja et al. and Zhao et al.: Targeting CD47 on human solid tumors. Proc Natl Acad Sci U S A 2012, 109:2844-2845.

43. Jaiswal S, Jamieson CH, Pang, WW, Park CY, Chao MP, Majeti R, Traver D, van Rooijen N, Weissman IL: CD47 is upregulated on circulating hematopoietic stem cells and leukemia cells to avoid phagocytosis. Cell 2009, 138:271-285.

44. Lindberg FP, Lublin DM, Telen MJ, Veile RA, Miller YE, Donis-Keller H, Brown EJ: Rhrelated antigen CD47 is the signal-transducer integrin-associated protein. J Biol Chem 1994, 269:1567-1570.

45. Gardai SJ, McPhillips KA, Frasch SC, Janssen WJ, Starefeldt A, Murphy-Ullrich JE, Bratton DL, Oldenborg PA, Michalak M, Henson PM: Cell-surface calreticulin initiates clearance of viable or apoptotic cells through trans-activation of LRP on the phagocyte. Cell 2005, 123:321-334.

46. Arfsten J, Leupold S, Bradtmoeller C, Kampen I, Kwade A: Atomic force microscopy studies on the nanomechanical properties of Saccharomyces cerevisiae. Colloid Surf B 2010, 79:284-290. 
47. Beningo KA, Wang YL: Fc-receptor-mediated phagocytosis is regulated by mechanical properties of the target. J Cell Sci 2002, 115: 849-856.

48. Lam WA, Rosenbluth MJ, Fletcher DA: Chemotherapy exposure increases leukemia cell stiffness. Blood 2007, 109:3505-3508.

49. Cross SE, Jin YS, Rao J, Gimzewski JK: Nanomechanical analysis of cells from cancer patients. Nat Nanotechnol 2007, 2:780-783.

50. Merkel TJ, Jones SW, Herlihy KP, Kersey FR, Shields AR, Napier M, Luft JC, Wu H, Zamboni WC, Wang AZ, Bear JE, DeSimone JM: Using mechanobiological mimicry of red blood cells to extend circulation times of hydrogel microparticles. Proc Natl Acad Sci U S A 2011, 108:586-591.

51. Mebius RE, Kraal G: Structure and function of the spleen. Nat Rev Immuno/ 2005, 5:606616.

52. Deplaine G, Safeukui I, Jeddi F, Lacoste F, Brousse V, Perrot S, Biliqui S, Guillotte M, Guitton C, Dokmak S, Aussilhou B, Sauvanet A, Cazals Hatem D, Paye F, Thellier M, Mazier D, Milon G, Mohandas N, Mercereau-Puijalon O, David PH, Buffet PA: The sensing of poorly deformable red blood cells by the human spleen can be mimicked in vitro. Blood 2011, 117:88-95.

53. Champion JA, Mitragotri S: Role of target geometry in phagocytosis. Proc Natl Acad Sci USA 2006, 103:4930-4934. 
A

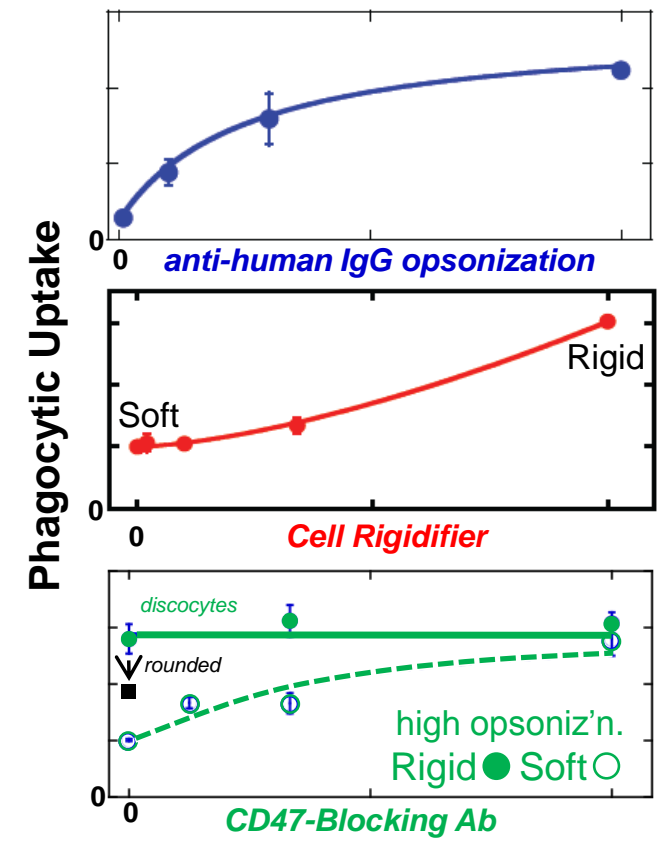

B

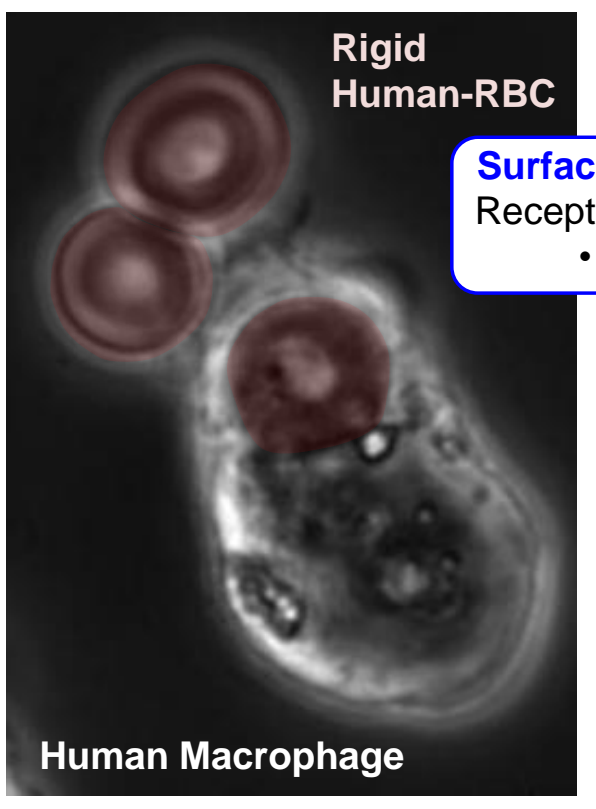

C fast

Interactions Pair: - Fc $\gamma R$ - IgG

Intracellular Signaling

Acto-myosin Activation

Physical Properties of Target

- Stiffness: Rigid

- Shape: Concave

\section{slow}

Surface Interaction

Receptor-Ligand Pair: - SIRPA-CD47

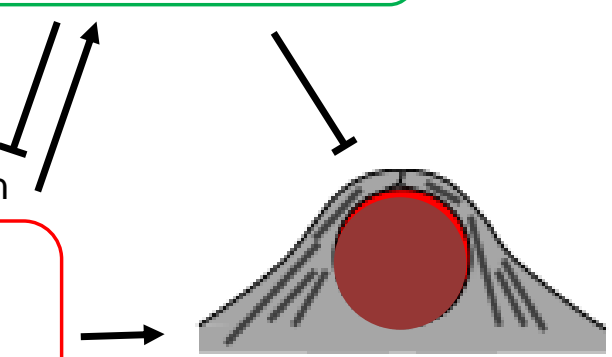

Phagocytic Uptake

Figure 1. Target physical properties and molecular interactions at the cell surface determine the efficiency of human RBC engulfment by human Macrophages (adapted from Sosale 2015). (A) Phagocytosis increases with IgG opsonization and with crosslinker rigidification of RBCs. Phagocytosis of rigid, opsonized RBCs is independent of CD47 inhibition in contrast to 'Soft' native RBCs whose uptake is enhanced by a CD47-blocking antibody. A 'sphering' treatment that gives a rounded and rigid RBC shows reduced uptake relative to discocytes. (B) Timelapse images of rigidified RBC discocytes shows rapid engulfment and lack of deformation by the macrophage.

(C) Surface interactions combine kinetically with physical properties of a candidate target in the calculus that determines phagocytic uptake. 